Engineering

Manuscript Number:

Title: Numerical investigation of vortex-induced motions of a pairedcolumn semi-submersible in currents

Article Type: VSI: Marine CFD

Keywords: Vortex-induced motions; paired-column semi-submersible; detached-eddy simulation; overset grid; naoe-FOAM-SJTU solver

Corresponding Author: Professor Decheng Wan, Ph.D.

Corresponding Author's Institution: Shanghai Jiao Tong University

First Author: Weiwen Zhao, PhD

Order of Authors: Weiwen Zhao, PhD; Lu Zou, PhD; Decheng Wan, Ph.D.; Zhiqiang $\mathrm{Hu}$, Dr

Abstract: Vortex-induced motions (VIM) is becoming a noteworthy issue for column-stabilized floating platforms, mainly due to its substantial fatigue damage to risers and mooring system. The VIM of deep-draft semisubmersible is more complex than single column floaters because of the wake interference between columns, as well as the considerable yaw motions. In the present work, a numerical approach for simulating VIM of deep-draft semi-submersible is proposed. Specifically, detached-eddy simulation is used for turbulence modeling and dynamic overset grid technique is used for moving objects. Simulations for stationary drag and VIM of a model-scale paired-column semi-submersible are conducted with the proposed approach. The numerical results are compared with experimental data. Transverse, in-line and yaw motions are allowed during VIM simulations and are further analyzed in frequency domain by Fast Fourier Transform (FFT). Different VIM characteristics are observed at different current velocities. The work done by each component of the structure is also discussed. Flow visualizations are presented for better understanding of the wake interferences during VIM. The accuracy and reliability of the current numerical approach is assessed.

Suggested Reviewers: Shixiao Fu PhD

Professor, Shanghai Jiao Tong University

shixiao.fulajtu.edu.cn

Prof. Shixiao Fu is expert of VIM analysis.

Changhong Hu PhD

Professor, Kyushu University, Japan

hu@riam.kyushu-u.ac.jp

Prof. Changhong $\mathrm{Hu}$ is famous expert of CFD on marine hydrodynamics.

Qing Xiao PhD

Reader, Strathclyde University, UK

qing.xiaodstrath.ac.uk

Dr. Qing Xiao is expert of CFD on and FSI and marine hydrodynamics. 

Dear Atilla and Editor,

It is our pleasure to submit our recent finished paper with title "Numerical investigation of vortex-induced motions of a paired-column semi-submersible in currents" to Ocean Engineering. Thanks for your kind review and consideration of publication in Ocean Engineering.

Best regards,

Decheng

$--$

Prof. Dr. Decheng Wan

Chair Professor of Chang Jiang Scholars

Head of Computational Marine Hydrodynamics Lab

School of Naval Architecture, Ocean and Civil Engineering

Shanghai Jiao Tong University (SJTU)

Dongchuan Road 800

Shanghai 200240

China 
Highlights:

1. Perform numerical investigations of VIM of deep-draft paired-column semi-submersible.

2. Present spectral analysis of motion responses by Fast Fourier Transform (FFT).

3. Pontoons are responsible for mitigating VIM.

4. Reveal the synchronized vortex shedding around upstream columns in lock-in range.

5. Flow reattachments are responsible for amplifying VIM. 


\title{
Numerical investigation of vortex-induced motions of a paired-column semi-submersible in currents
}

\author{
Weiwen Zhao ${ }^{1}$, Lu Zou ${ }^{1}$, Decheng Wan ${ }^{1 *}$, Zhiqiang $\mathrm{Hu}^{2}$ \\ ${ }^{1}$ State Key Laboratory of Ocean Engineering, School of Naval Architecture, Ocean and Civil \\ Engineering, Shanghai Jiao Tong University, Collaborative Innovation Centre for Advanced Ship \\ and Deep-Sea Exploration, Shanghai, China \\ ${ }^{2}$ School of Marine Science \& Technology, Newcastle University, Newcastle upon Tyne, UK \\ *Corresponding author: dcwan@sjtu.edu.cn \\ Abstract
}

\begin{abstract}
Vortex-induced motions (VIM) is becoming a noteworthy issue for column-stabilized floating platforms, mainly due to its substantial fatigue damage to risers and mooring system. The VIM of deep-draft semi-submersible is more complex than single column floaters because of the wake interference between columns, as well as the considerable yaw motions. In the present work, a numerical approach for simulating VIM of deep-draft semi-submersible is proposed. Specifically, detached-eddy simulation is used for turbulence modeling and dynamic overset grid technique is used for moving objects. Simulations for stationary drag and VIM of a model-scale paired-column semi-submersible are conducted with the proposed approach. The numerical results are compared with experimental data. Transverse, in-line and yaw motions are allowed during VIM simulations and are further analyzed in frequency domain by Fast Fourier Transform (FFT). Different VIM characteristics are observed at different current velocities. The work done by each component of the structure is also discussed. Flow visualizations are presented for better understanding of the wake interferences during VIM. The accuracy and reliability of the current numerical approach is assessed.
\end{abstract}

Keywords: Vortex-induced motions; paired-column semi-submersible; detached-eddy 
simulation; overset grid; naoe-FOAM-SJTU solver

\section{Introduction}

Modern offshore structures are often designed to have deep draft stabilized columns and low gravitational center in order to suppress the wave-induced motions, especially for heave motions.

These column structures are subject to motions that are induced by the periodical fluctuation forces and vortex shedding when currents velocities exceed a few knots. The term vortex-induced motions (VIM) is coined to describe the phenomenon as the motions are caused by vortices. VIM is a matter of high complexity, mainly due to the high Reynolds numbers turbulent flows around floating structure with complex geometry, as well as six-degrees-of-freedom motions that are determined by various kinds of forces such as hydrodynamic forces, mooring forces, gravitational forces, not to mention the interaction between the fluids and structures. VIM is similar to vortex-induced vibrations (VIV). The latter generally represents the high frequency vibrations of rigid or flexible cylinders with large aspect ratio, such as risers and cables. In contrast, VIM describes the much longer period motions of large volume offshore structures, such as Spars, monocolumn, semi-submersibles, TLPs and buoys. In the oil drilling production environment, the floating structures are moored with mooring lines. The floating structure and mooring lines can be treated as a spring-mass system. When the frequency of external excitation (vortex shedding or transverse hydrodynamic force) is at or near the structural natural frequency of the system in still water, the storage of vibrational energy increases rapidly which produces large amplitude oscillations up to one diameter of column. Unlike stationary cylinder whose shedding frequency is 
proportional to velocity, the shedding frequency of spring-supported cylinder is locked in one natural frequency of the cylinder. This so-called "lock-in" phenomenon greatly accelerates the fatigue failure of mooring and risers system and reduces the service life span of offshore platforms [1].

There have been plenty of studies on VIM for various kinds of offshore platforms, most of which are performed by means of model tests in towing tanks and numerical simulations with computational fluid dynamic (CFD). Geometric similitude is important for model test and is achieved by scaling not only model geometry but also appurtenance from prototype accurately. Another important aspect is dynamic similitude which requires the properly scale of natural periods, mass ratio and reduced velocities [2]. It is well known that it's impossible to keep both Reynolds number scaling and Froude number scaling for hydrodynamic model testing of offshore structures. VIM model tests apply Froude scaling for hydrodynamic similitude due to the speed limitation of towing facilities. The scaling effect brought by Reynolds number has been addressed by Roddier et al. [3]. They conducted a series of model tests for the hard tank part of a Truss Spar model in three different scale ratios (three different Reynolds regimes) and concluded that there are little differences between sub-critical and super-critical regimes, which means Froude scaling in terms of geometric and dynamic similitude is applicable for VIM. 
remarkable accuracy. Most of the simulations were carried out with commercial CFD software, such as the finite element solver AcuSolve $[4,7,8,11]$ and the finite volume solvers Star-CCM+[5,7] and Fluent [7,8]. Exceptions are Lee et al. [6] and Chen and Chen [9] who investigated the round-corner effect and scale effect of VIM of a deep-draft semi-submersible at model scale and full scale using an in-house Finite-Analytic Navier-Stokes (FANS) code which solves Reynolds-Average Navier-Stokes (RANS) equations in curvilinear body-fitted coordinate system with overset structured grid capability. Rosetti et al. [12] presented numerical simulations of VIM of a semi-submersible with circular columns in 0 and 45 degrees current heading by using ReFRESCO which is an in-house viscous-flow CFD code that solves multiphase unsteady incompressible flows using Navier-Stokes equations. Recently, the open source CFD software OpenFOAM raises as a popular CFD software in both academia and industry due to its flexible and extensible design and good source code quality. It's easy and convenient to implement customized functionality based on the framework. Zhao et al. [13] simulated VIM of a Spar platform in uniform currents using an in-house solver naoe-FOAM-SJTU which is developed based on OpenFOAM. The effectiveness of helical strake on suppressing VIM was discussed. Kara et al. [10] calculated VIM of a paired-column semi-submersible using the free, open source CFD software OpenFOAM. They implemented an in-house 6 degree-of-freedom (6DoF) solver with nonlinear coupling of accelerations and velocities to solve the motions. The $6 \mathrm{DoF}$ solver has an interface for generalized external forces such as spring forces. They also highlighted the key aspects of CFD methodology for VIM simulations and concluded that the detached-eddy simulation (DES) is a powerful turbulence model in estimating response amplitude and periods. 
In the present paper, the CFD simulation of a paired-column semi-submersible with eight-columns and squared-pontoon hull configuration is performed using the in-house CFD code naoe-FOAM-SJTU. To resolve the turbulent wake structures and predict the wake interference between columns and pontoons, a DES turbulence model (SST-DDES) is employed. An unstructured overset grid approach is adopted to avoid mesh distortion and support the arbitrary large movements of hull. The objective of the present study is to demonstrate the validity of the current numerical approach to VIM problems. The approach is discussed and applied to a problem of VIM for semi-submersibles. Results are compared with experiments and simulation results from the RPSEA 5404 project [14].

\section{Mathematical and numerical methods}

A finite volume CFD solver naoe-FOAM-SJTU $[15,16]$ is used to perform all simulations. The naoe-FOAM-SJTU was initially developed based on the open source platform OpenFOAM version 2.0.1. It was derived from interDyMFoam (a standard solver from OpenFOAM) with an in-house 6DoF solver based on Euler angles and a wave generation and absorption module for various types of regular and irregular waves common in marine and ocean engineering. Furthermore, the dynamic overset capability was implemented into the solver in coupled with Suggar [17] to perform large amplitude hull motions. Recently, the solver was upgraded to OpenFOAM version 3.0 and use Suggar++ [18], an improved version of Suggar, to compute domain connectivity information (DCI), and to connect fields solutions among multiple overset mesh blocks. The naoe-FOAM-SJTU has been validated against a majority of steady and unsteady problems [19-21]. In this study, the free surface was neglected due to the low Froude number 
110 condition

128 where $k$ is the turbulent kinetic energy and $\omega$ is the specific dissipation rate. The turbulence

\subsection{Governing equations and turbulence modeling} for turbulent flow in vectorial form are written as and $v_{t}$ is the turbulent eddy viscosity. transport equations for SST are given as

The flow is treated as single-phase and incompressible. The continuity and momentum equations

$$
\begin{gathered}
\nabla \cdot \mathbf{U}=0 \\
\frac{\partial \mathbf{U}}{\partial t}+\nabla \cdot\left(\mathbf{U}-\mathbf{U}_{g}\right) \mathbf{U}=-\frac{1}{\rho} \nabla p+\nabla \cdot\left(v_{e f f} \nabla \mathbf{U}\right)+(\nabla \mathbf{U}) \cdot \nabla v_{e f f}
\end{gathered}
$$

where, $\mathbf{U}$ is the fluid velocity and $\mathbf{U}_{g}$ is the grid velocity, $p$ is the pressure and $\rho$ is the fluid density. The effective viscosity is defined as $v_{\text {eff }}=v+v_{t}$, where $v$ is the molecular viscosity

The delayed DES (DDES) formulation of $k-\omega$ shear stress transport (SST) model is employed for turbulence modeling. The SST model is a blended $k-\omega / k-\varepsilon$ model which has been proven to be robust and accurate for turbulent flows around complex geometries in industry. DDES is a hybrid RANS/LES method which combines the best practice of RANS and LES in a single solution strategy. In this study, SST-DDES solves flow field using $k-\omega$ SST model in the near wall regions and converts to LES subgrid-scale model in other regions after flow separation. The

$$
\begin{gathered}
\frac{\partial k}{\partial t}+\nabla \cdot(\mathbf{U} k)=\tilde{G}-\frac{k^{3 / 2}}{l}+\nabla \cdot\left[\left(v+\alpha_{k} v_{t}\right) \nabla k\right] \\
\frac{\partial \omega}{\partial t}+\nabla \cdot(\mathbf{U} \omega)=\gamma S^{2}-\beta \omega^{2}+\nabla \cdot\left[\left(v+\alpha_{\omega} v_{t}\right) \nabla \omega\right]+\left(1-F_{1}\right) C D_{k \omega}
\end{gathered}
$$

129 length scale $l$ in SST model is defined as $l=l_{\text {RANS }}=\frac{k^{3 / 2}}{\beta^{*} k \omega}$. SST-DDES modifies the length 


$$
l_{D D E S}=l_{R A N S}-f_{d} \max \left(0, l_{R A N S}-C_{D E S} \Delta\right)
$$

where $f_{d}$ is empiric blending function defined as

Here $S$ and $\Omega$ are strain rate and vorticity tensor invariants, $\kappa=0.41$ is the von Karman $(\phi, \theta, \psi)$ (roll, pitch, yaw) of the rigid body in Earth frame. The transformation of linear velocity $(u, v, w)$ angular velocity $(p, q, r)$ in body-fixed frame between the two coordinate frames can be constant, $d_{w}$ is wall distance. The blending function $f_{d}$ is zero inside boundary layer to deactivate the DES limiter and protect the boundary layer from earlier separation.

\subsection{DoF rigid body solver}

There has already been a standard 6DoF rigid body motion solver in OpenFOAM. In this 6DoF solver, the rigid body motion state is described by quaternions. While in the marine and ocean engineering context, an Euler angle description of motions for marine structures is preferred for convenience. Therefore, an in-house $6 \mathrm{DoF}$ solver based on Euler angles for marine hydrodynamic applications was adopted in naoe-FOAM-SJTU [15]. Two coordinates, namely inertia and non-inertia frame, are used for solving the motion equations of the rigid body. The inertial frame or Earth frame is fixed to Earth or moves at a constant speed with respect to the Earth. The non-inertial frame or body-fixed frame is fixed on the rigid body and translates and rotates according to the motions of rigid body with respect to the inertial system. The two coordinate frames are related to each other by the positions $(x, y, z)$ (surge, sway, heave) and Euler angles 
152 applying Newton's $2^{\text {nd }}$ law and governed by the following equations in body-fixed coordinate

frame

$$
\left\{\begin{array}{c}
\dot{u}=X / m+v r-w q+x_{g}\left(q^{2}+r^{2}\right)-y_{g}(p q-\dot{r})-z_{g}(p r+\dot{q}) \\
\dot{v}=Y / m+w p-u r+y_{g}\left(r^{2}+p^{2}\right)-z_{g}(q r-\dot{p})-x_{g}(q p+\dot{r}) \\
\dot{w}=Z / m+u q-v p+z_{g}\left(p^{2}+q^{2}\right)-x_{g}(r p-\dot{q})-y_{g}(r q+\dot{p}) \\
\dot{p}=\frac{1}{I_{x}}\left\{K-\left(I_{z}-I_{y}\right) q r-m\left[y_{g}(\dot{w}-u q+v p)-z_{g}(\dot{v}-w p+u r)\right]\right\} \\
\dot{q}=\frac{1}{I_{y}}\left\{M-\left(I_{x}-I_{z}\right) r p-m\left[z_{g}(\dot{u}-v r+w q)-x_{g}(\dot{w}-u q+v p)\right]\right\} \\
\dot{r}=\frac{1}{I_{z}}\left\{N-\left(I_{y}-I_{x}\right) p q-m\left[x_{g}(\dot{v}-w p+u r)-y_{g}(\dot{u}-v r+w q)\right]\right\}
\end{array}\right.
$$

where $m$ is the mass and $I_{x}, I_{y}, I_{z}$ are the moments of inertia around centre of rotation,

$X, Y, Z, K, M$ and $N$ are surge, sway, heave forces and roll, pitch, and yaw moments. By solving

the $6 \mathrm{DoF}$ motion equations, the linear and angular accelerations in body-fixed frame are obtained.

158 The linear and angular velocities in body-fixed frame are obtained by integral acceleration over time and then they are transformed to Earth system. Finally, the translations and rotations increments are obtained by integral velocities over time and they are used in the next stage for grid external forces (such as mooring forces or constant force).

\subsection{Mooring system}

164 Mooring system consists of several mooring lines. Each mooring line is anchored to a fixed point

165 at one end and attached to the moving body at the other end. The framework of mooring system

166 follows the object-oriented (OO) design approach and OpenFOAM data structures. An abstract

167 base class named mooringLine is designed for describing general mooring line. This class

168 provides interfaces such as computing forces and moments (around centre of rotation), updating

169 mooring line shapes. These member functions will be implemented in derived classes for different 
types of mooring lines. Currently supported mooring line types include linearSpring, catenary, PEM (piecewise extrapolation method) and $L M M$ (lumped mass method). It is easy to extend the mooring system and add new mooring line types based on the current framework thanks to the OO design. Finally, a wrapper PtrList<mooringLine> is used for the whole mooring system that consisting all kinds of mooring lines, as shown in Figure 1. The solution procedure of mooring system is as summarized follows: compute mooring forces and moments and add them as external excitation to the rigid body motion equations; solve the $6 \mathrm{DoF}$ motion equations and update motion state for rigid body; update the mooring line shape for current time step and step forward to next time step. In the present study, all mooring lines are treated as linear springs.

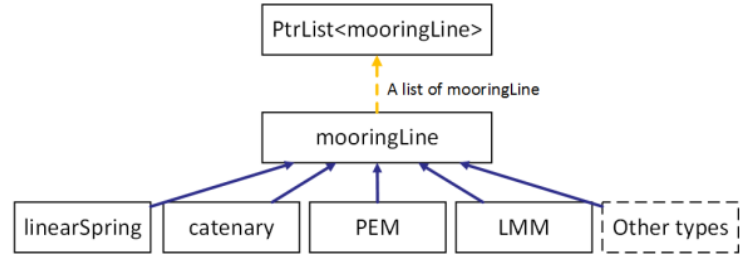

Figure 1: Framework of mooring system module

\subsection{Overset grid}

The naoe-FOAM-SJTU uses an overset grid system to solve the flow field. This is achieved by the combination of the grid assembly Suggar++ [18] and OpenFOAM. Details of the coupling strategy can be referred to Shen's work [16] and only a brief introduction is presented here.

A parallel scheme is archived in naoe-FOAM-SJTU by running OpenFOAM and Suggar++ processors simultaneously. Suggar++ is responsible for computing DCIs that contain cell type information (e.g., active, hole, orphan, fringe and donor) and interpolating weighting factors. OpenFOAM was responsible for solving fluid, computing forces and motions of the rigid body, updating mesh. DCIs are sent from Suggar++ to OpenFOAM processors with MPI. Currently, 
190 Suggar++ does not recognize OpenFOAM mesh format, a copy of overset mesh will be converted

191 from OpenFOAM format to Suggar++ supported format before computation. In other words, the

192 solver keeps two separated grid instance, one for OpenFOAM and one for Suggar++. The

193 Suggar++ grid is updated with the rigid body motion state obtained by OpenFOAM.

\subsection{Solution strategy}

195 The overall solution strategy is illustrated in Figure 2. At the beginning of simulation, OpenFOAM

196 read meshes, boundary conditions and initial conditions for initialization. After that OpenFOAM

197 receives DCIs from Suggar++, performs PIMPLE loop to obtain pressure and velocity and solves

198 transport equations for turbulence quantities. Then pressure, viscous and mooring forces are

199 computed and motions are predicted. The motion data will be sent to Suggar++ to update grids

200 used by Suggar++. The DCIs in Suggar++ processor is decomposed by OpenFOAM's domain

201 decomposition and cell distribution information and will be sent to each OpenFOAM processor.

202

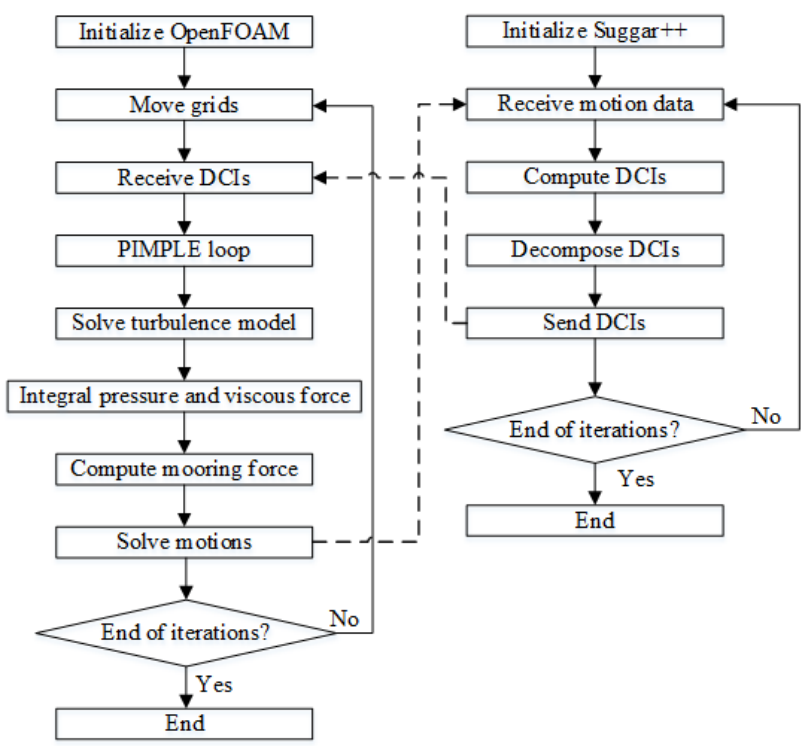

Figure 2: Flow chart of the whole solution strategy

\section{Simulation design}



be found in Table 1.

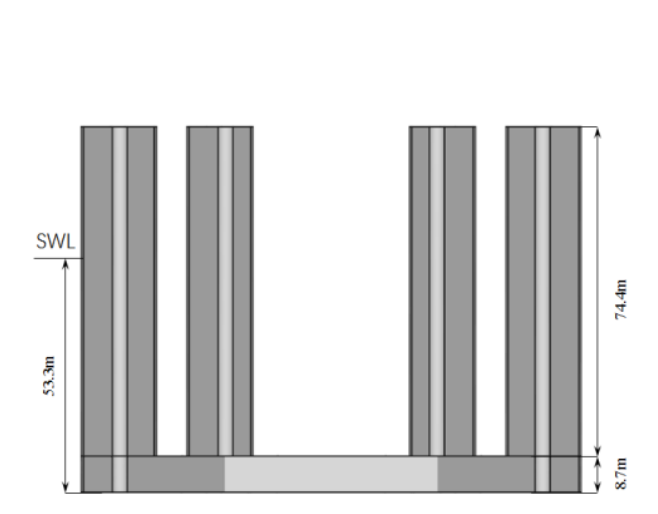

draft is $53.3 \mathrm{~m}$. Columns are divided into outer column (OC) and inner column (IC). OCs are connected to ICs at four corners via pontoon. Both OC and IC have rectangular section, while sizes are different, i.e., $14 \times 13.4 \mathrm{~m}$ and $14 \times 10.4 \mathrm{~m}$, respectively. The base gap between OC and IC is 20.4m, and tensioner stroke is $8.5 \mathrm{~m}$. The model scale for PC Semi in the current study is 1:54. Details about the main particular of the PC Semi geometry in both full-scale and model-scale can

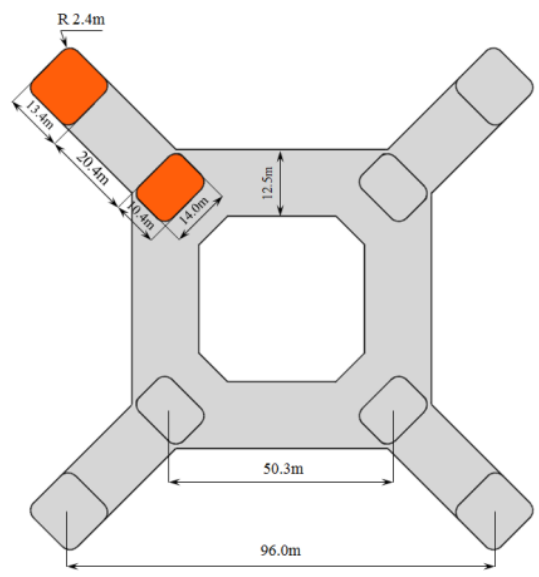

Figure 3: Side view and top view of the prototype geometry

Table 1: Main particulars of the prototype and model

\begin{tabular}{llll}
\hline Name & Notation (unit) & Prototype & Model \\
\hline Overall width & $B(\mathrm{~m})$ & 113.4 & 2.1 \\
Draft & $T(\mathrm{~m})$ & 53.3 & 0.987
\end{tabular}


44.6

0.826

\begin{tabular}{|c|c|c|c|}
\hline Outer column size & $L_{O C} \times W_{O C}(\mathrm{~m})$ & $13.4 \times 14$ & $0.248 \times 0.259$ \\
\hline Outer column characteristic & $D(\mathrm{~m})$ & 19.4 & 0.36 \\
\hline \multicolumn{4}{|l|}{ length } \\
\hline Inner column size & $L_{I C} \times W_{I C}(\mathrm{~m})$ & $10.4 \times 14$ & $0.192 \times 0.259$ \\
\hline Inner column characteristic & $d(\mathrm{~m})$ & 17.4 & 0.32 \\
\hline \multicolumn{4}{|l|}{ length } \\
\hline Center-to-center distance of & $S_{O C}(\mathrm{~m})$ & 96.0 & 1.78 \\
\hline \multicolumn{4}{|l|}{ outer column } \\
\hline Center-to-center distance of & $S_{I C}(\mathrm{~m})$ & 50.3 & 0.93 \\
\hline \multicolumn{4}{|l|}{ inner column } \\
\hline Pontoon height & $P(\mathrm{~m})$ & 8.7 & 0.16 \\
\hline Pontoon width & $L_{p}(\mathrm{~m})$ & 12.5 & 0.23 \\
\hline
\end{tabular}

218 The case conditions consist of two parts: the stationary drag and the VIM simulation. In stationary

219 drag simulation, the semi-submersible is fixed and not allowed to move. Static overset grid is used

220 for stationary drag simulation. In this approach, as the hull is stationary and grids do not move,

221 DCIs are computed at the beginning of the simulation and do not need to be updated in the

222 following time steps. VIM simulation utilizes dynamic overset grid to perform hull boundary

223 movement. In every time step, the DCIs are reinitialized automatically to update the hole-cutting

224 geometry. Motions in horizontal plane (e.g., surge, sway and yaw) are allowed during VIM 
225 simulation. eliminate effect from boundaries at two lateral sides, downstream and bottom.

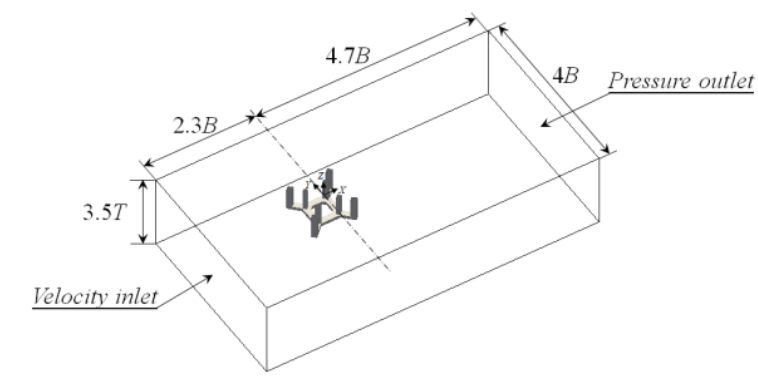

\subsection{Coordinate system and grids}

A right-handed Cartesian coordinate system $O-X Y Z$ is used in the simulations. The origin $O$ is located at the center point of hull geometry on the surface water line. $X$-axis coincides with current direction and points towards the downstream. $Y$-axis points to the transverse direction (starboard) that perpendicular to current and Z-axis points upwards.

The computational domain is set as $7 B \times 4 B \times 3.5 T$ (length $\times$ width $\times$ depth) for all simulations as shown in Figure 4. Here, $B$ is the overall width and $T$ is the draft of the hull. In previous studies of semi-submersible VIM, the computational domain size are slightly different. Kim et al. [4] used a domain of $14 B \times 12 B \times 4.5 T$. A $27 B \times 18 B \times 6 T$ domain was adopted by Tan et al. [5], and $18 B \times 12 B \times 6 T$ by Liu et al. [22]. Compared with these domains, smaller domain size is also acceptable. For example, Lee et al. [6] numerically studied VIM of a deep-draft semi-submersible using computational domains of $6 B \times 4.5 B \times 2.8 T$ and $5 B \times 4 B \times 2.2 T$. A $9 B \times 6 B \times 3 T$ domain was selected by Liang and Tao [23] in their studies of vortex shedding process of flow around a deep-draft semi-submersible. It is reasonable that the current domain size is large enough to

Figure 4: Computational domain and boundaries 
243 A constant and uniform flow condition is employed for all simulations. The boundary condition

244 for velocity is set as $(U, 0,0)$ ( $U$ the current velocity) at inlet and zero gradient at outlet. As for

245 pressure, a zero gradient boundary condition and zero value is set for inlet and outlet, respectively.

246 Symmetry planes are specified for two lateral sides and bottom boundaries. Besides, symmetric

247 boundary condition is also applied for top due to the ignorance of free surface effect at low Froude

248 number conditions. For hull surface of the PC Semi, a no-slip boundary condition is prescribed

249 which assigns the velocity to $\boldsymbol{U}_{\text {wall }}$ and the pressure to zero normal gradient.

250 An unstructured polyhedral multi-block overset grid system is used throughout the present study.

251 The grid system consists of two blocks, namely the background and hull grid, which are generated

252 individually and then assembled into a single mesh. The background mesh block is hexahedral and

253 has a uniform grid spacing. The hull mesh block is based on predominantly Cartesian cut-cell

254 approach and has a same initial base grid size with background mesh block to avoid orphans when

255 performing overset DCI calculation. The near hull and wake regions are refined in the hull mesh

256 block in order to capture the boundary layers and wake structures induced by flow separations.

257 Four different levels of refinement zones are utilized to archive higher accuracy in critical regions.

258 In the vicinity of columns and pontoons, 10 prism cell layers are applied to hull boundary to

259 capture the boundary layer development. For all cases, the non-dimensioned wall distance of the

260 first layer satisfies $y+<1$, which makes sure the first layer cells are located in the viscous sublayer.

261 An example mesh can be found in Figure 5. 

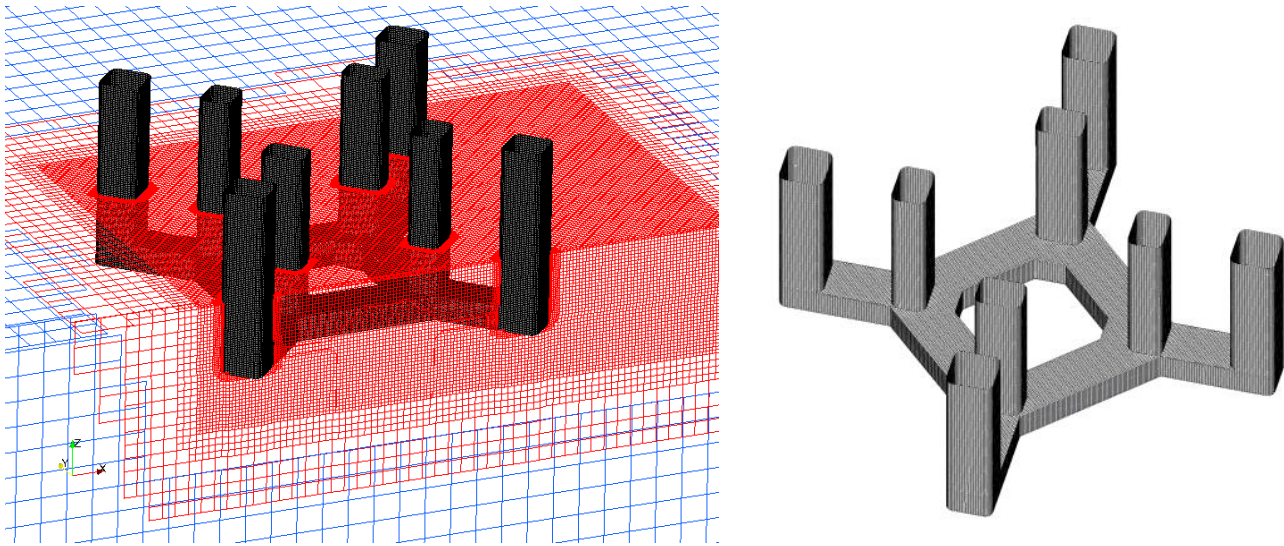

Figure 6: Overset mesh assembly and hull surface mesh

convection term in momentum equation. For turbulent quantities convection terms, a second-order

Total Variation Diminishing (TVD) limited linear scheme is used. The merged PISO-SIMPLE

(PIMPLE) algorithm is used for pressure-velocity decoupling.

\subsection{Mooring stiffness}

273 The most vital part of mooring system is not the configuration of the mooring lines but the

274 equivalent restore stiffness provided by the mooring system. As the stiffness has direct influence

275 on the moored floating body's natural period which significantly affect the VIM response

276 characteristics. To make the comparison with experimental data meaningful, one must verify the

277 effective stiffness before VIM simulation. In the experiments [24], the model was equipped with

278 frictionless air bearings that slide along a horizontal plate. This air bearing system allows the 
279 model moving freely in the horizontal plane. Meanwhile, the vertical motions are constrained.

280 Table 2 lists the mass and stiffness properties of the hull and mooring system from experiment.

281 Table 2: Main particular for mass and stiffness system at model scale

\begin{tabular}{lll}
\hline Name & Value & Dimensions \\
\hline Mass & 490.2 & $\mathrm{~kg}$ \\
Radius of gyration & 0.77593 & $\mathrm{~m}$ \\
Transverse stiffness & 173.98 & $\mathrm{~N} / \mathrm{m}$ \\
Yaw stiffness & 5.23 & $\mathrm{Nm} / \mathrm{deg}$ \\
Transverse natural period & 15.45 & $\mathrm{~s}$ \\
Yaw natural period & 9.32 & $\mathrm{~s}$ \\
\hline
\end{tabular}

283 In the present numerical simulations, an equivalent horizontal mooring system is used. The

284 mooring system consists of four linear springs that distribute along positive and negative $X$-axis

285 and $Y$-axis. Figure 7 depicts the sketch of the mooring system. All spring are pretensioned and the

286 pretension should be large enough to ensure that the spring would not relax during VIM.

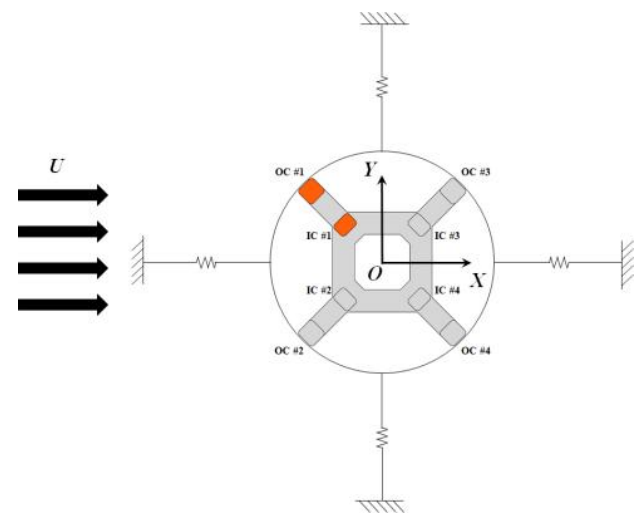

Figure 7: Schematic of the mooring system configuration 
289 To perform validation for stiffness, static offset tests and free decay tests are carried out in

290 sequence. In static offset tests, the hull is prescribed to move in $Y$-axis and rotate around $Z$-axis,

291 respectively, without solving flow field. Parameters such as stiffness and pretension of each spring

292 are adjusted to match the global horizontal and vertical (yaw) stiffness of model test. After static

293 offset test, the spring parameters are used for free decay tests. The free-decay tests allow hull

294 oscillating with a prescribed initial offset or velocity in the absence of inflow. Transverse and yaw

295 decay test are conducted separately to verify the natural transverse and yaw period of the mooring

296 system. The time histories and spectral analyses of free decay test are shown in Figure 8. The

297 deviations of natural period between CFD and EFD for transverse motion and yaw are $0.6 \%$ and

$2982 \%$, respectively, showing the correct equivalent linear and rotational stiffness are provided by the

299 current mooring configuration.
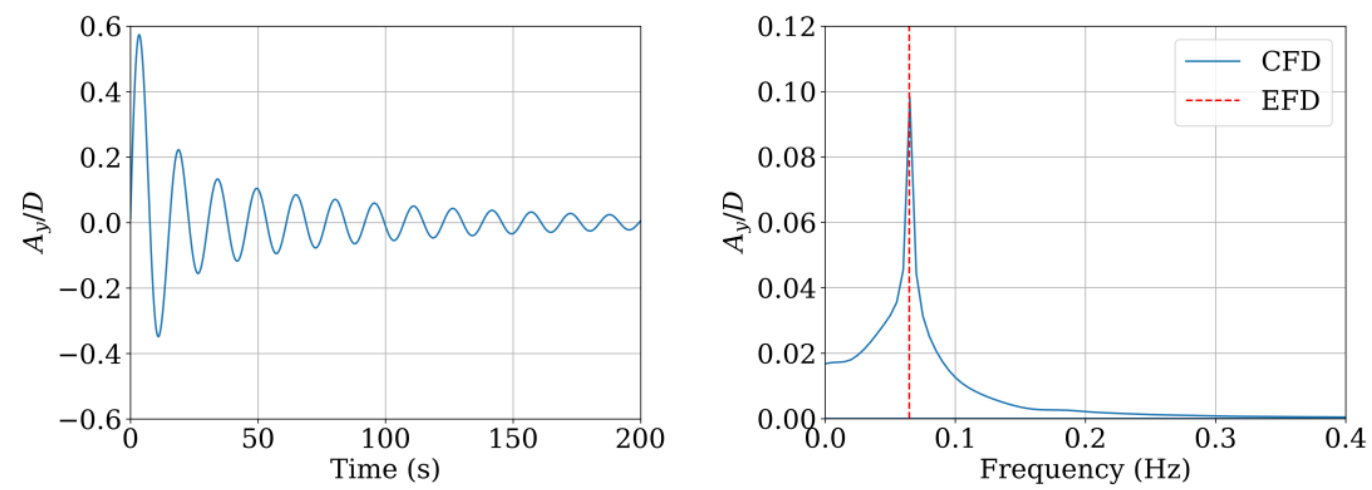

300
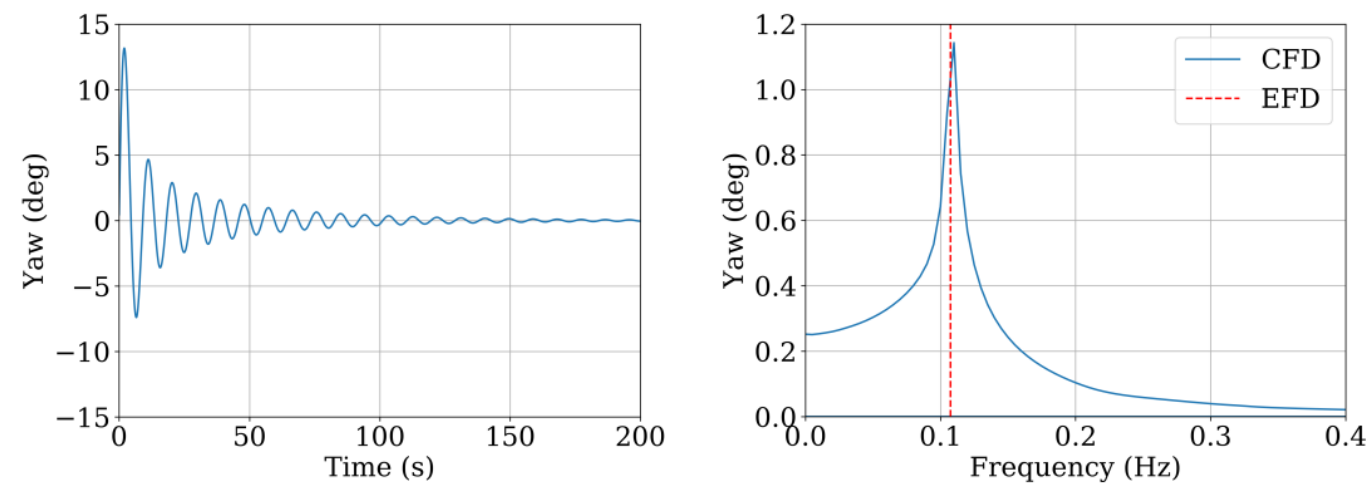
302 Figure 8: Time history and spectral analysis of transverse and yaw decay test

\section{Results and discussion}

\subsection{Stationary drag simulation}

In this case, the current velocity is $0.272 \mathrm{~m} / \mathrm{s}$. The time step is set to $0.02 \mathrm{~s}$ in all simulations including VIM simulations in the next section. Grid convergence study is performed on this case.

Three different mesh sizes are considered. Note that the overset mesh blocks used in the current study is unstructured grid. The background mesh block is uniform hexahedral mesh and is easy to refine in three directions of the Cartisian coordinate system. Contrary to background, the hull mesh block is obtained by cut and splitting cells in refinement region on an initial Cartisian grid.

Following the recommendation by Shen et al. $[15,16]$, to achieve consistent grid refinement ratio in three directions, the Cartisian grid is refined systematically by a factor. Table 3 lists the details of different cases in grid sensitivity study. Grid refinement ratio $r=1.4$ is selected for convergence study. Total grid number for coarse, medium and fine mesh are $1.04 \times 10^{6}, 2.53 \times 10^{6}$ and $6.25 \times 10^{6}$, respectively. The grid independent study shows that $S_{2}$ is fine enough to get reliable results at a relatively low computational cost and it is used in the following studies.

Table 3 Grid independent study for stationary drag simulation

\begin{tabular}{lllllll}
\hline Case & ID & \multicolumn{2}{l}{ No. of cells $\left(\times \mathbf{1 0}^{\mathbf{6}}\right)$} & & $\overline{\boldsymbol{C}_{\boldsymbol{D}}}$ & $\boldsymbol{C}_{\mathbf{L} \text { rms }}$ \\
\hline & & Total & Backgroud & Hull & & \\
Fine & $S_{1}$ & 6.25 & 0.29 & 5.96 & 0.673 & 0.021 \\
Medium & $S_{2}$ & 2.53 & 0.10 & 2.43 & 0.689 & 0.022 \\
Coarse & $S_{3}$ & 1.04 & 0.04 & 1.00 & 0.726 & 0.048 \\
\hline
\end{tabular}


Figure 9 shows the instantaneous flow visualizations presented by streamwise velocity contour and streamlines on two cut-planes at $z / H=-0.5$ and $z / H=-1$. It can be seen that wake interference between side-by-side OCs is insignificant. However, the wake behind front OC is strongly influenced by the front IC. Asymmetric wake is observed due to the speed up between front OC and IC. The wake interaction between front and rear ICs is clear due to the small spacing ratio $(L / d=2.89)$. As for front and rear OCs, the spacing ratio $(L / D=4.95)$ is large enough that the wake interference is trivial. Figure 9(b) shows the existence of pontoon suppresses vortex sheds from the front OC inner flank. The coherent vortical structures along column vertical direction is destroyed at the low end by pontoon. This may indicates the damp effect of pontoon on VIM

behavior.

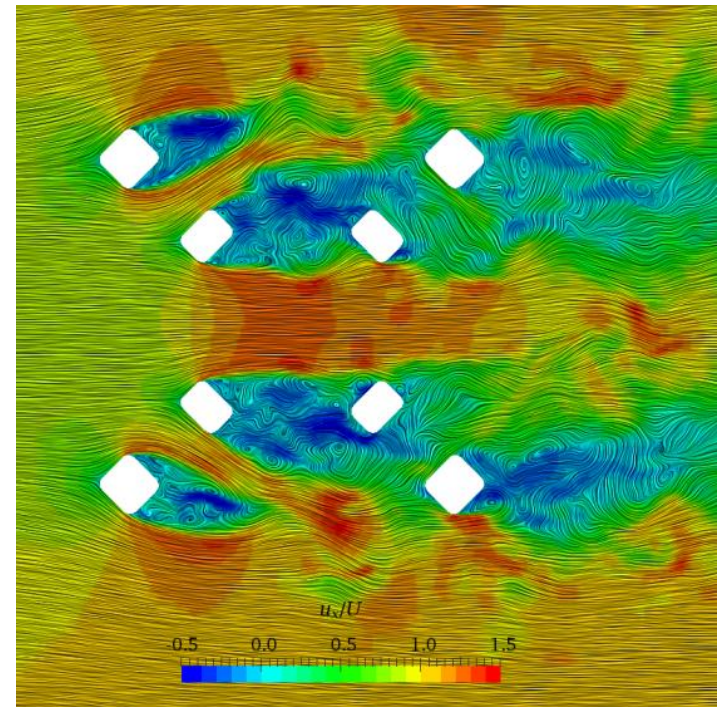

(a) $z / H=-0.5$

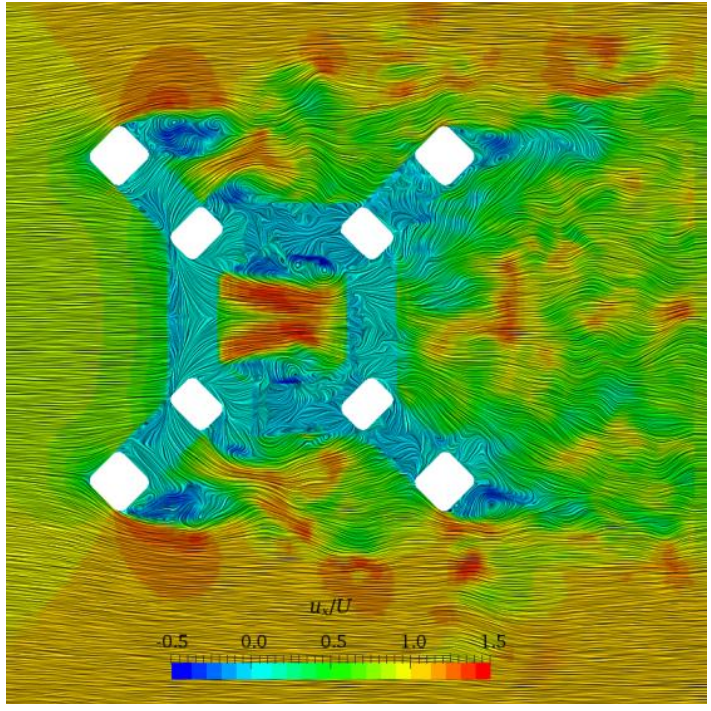

(b) $z / H=-1$

Figure 9: Instantaneous streamwise velocity contours and streamlines 
331 The non-dimensioned parameter reduced velocity is crucial to VIM response. It is defined as

$$
U_{r}=\frac{U T_{n}}{D}
$$

333 where $U$ is the current velocity or towing velocity, $T_{n}$ is the natural transverse period in still

334 water and $D$ is the characteristic length of the platform. In previous studies of semi-submersible 335 VIM, see for example [25-27], $D$ was the projected length of column section perpendicular to 336 flow direction. While in the current study, $D$ is $0.36 \mathrm{~m}$, which is the diagonal length of OC's 337 cross section. The definition is consistent with model test.

338 Five reduced velocities are considered. The model-scale current speeds range from $0.07 \mathrm{~m} / \mathrm{s}$ to present study. One is based on the root mean square $[28,7,24]$ and the other is based on standard deviation [25] of motion response time series. The corresponding definitions are listed below

$$
\begin{gathered}
\left(A_{x} / D\right)_{r m s}=\frac{\sqrt{2} R M S\left(A_{x}(t)\right)}{D},\left(A_{y} / D\right)_{r m s}=\frac{\sqrt{2} R M S\left(A_{y}(t)\right)}{D},(\operatorname{Yaw})_{r m s}=\sqrt{2} R M S(\text { yaw }(t)) \\
\left(A_{x} / D\right)_{s t d}=\frac{\sqrt{2} \sigma\left(A_{x}(t)\right)}{D},\left(A_{y} / D\right)_{s t d}=\frac{\sqrt{2} \sigma\left(A_{y}(t)\right)}{D},(\operatorname{Yaw})_{s t d}=\sqrt{2} \sigma(\operatorname{yaw}(t))
\end{gathered}
$$

Where RMS and $\sigma$ are the root mean square and standard deviation from motion time series, respectively, $A_{x}(t), A_{y}(t)$ and $y a w(t)$ are time histories for in-line, transverse and yaw motions, respectively. To better representing the VIM characteristics, the transition stage at the beginning of VIM response is eliminated for all simulations when performing statistical analysis and spectral analysis.

The nominal response in transverse direction is plotted in Figure 10. The black hollow square and circle represent the experimental data and CFD results taken from [24]. It's worth noting that the 
results at $U_{r}=5$ from model test have large dispersion for three repeated runs. In CFD simulations,

363 it takes longer time (40 dimensionless time step) for this particular case to reach pseudo steady state (quasi-sinusoidal transverse motion). This can be interpreted as the beginning of lock-in range. The vibrational energy stored in the system is increasing slowly at this condition. This was also found by Chen and Chen [9] in their CFD simulations of a deep draft semi-submersible. They stated that for a rounded-corner column semi-submersible at $U_{r}=6$ for model scale and at $U_{r}=4.4$ for prototype, a thousand more time steps is needed to reach the nominal amplitudes. As the current velocity increases, the vibrational energy increases more rapidly and the time to reach nominal amplitude decreases. The discrepancies between present CFD and AcuSolve is rather small. While both CFD results deviate from EFD with variation no more than $15 \%$. Nevertheless, it is reasonable that the current approach of VIM simulation can be considered reliable.

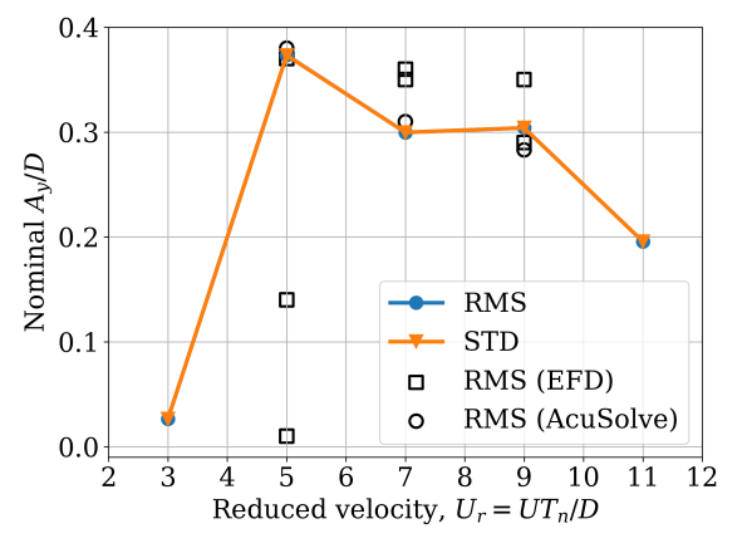

Figure 10: Nominal response of transverse motion

365 The RMS and STD are complete coincidence, meaning the average position of transverse motion is near 0 . When reduced velocity is small $\left(U_{r}=3\right)$, the nominal transverse motion response is rather

367 small (about 0.02). As the reduced velocity increases, the nominal response increases promptly, 368 suggesting a synchronized behavior in transverse motions $\left(5 \leq U_{r} \leq 9\right)$. The maximum amplitude is 
up to 0.37 and occurs at the beginning of lock-in range $\left(U_{r}=5\right)$.

370

371

Figure 11 shows the nominal response in in-line direction. RMS represents the average offset to origin and STD represents the fluctuation of VIM in in-line direction. For immersed structure exposed to current, the overall drag on structure increases with the current velocity increases. The offset between equilibrium position and origin becomes larger. This explains the increasing RMS of in-line response. Compared with transverse motion, the STD of in-line motion is small, which indicates much smaller fluctuation of in-line response. In addition, larger STD values are found at higher reduced velocities $\left(9 \leq U_{r} \leq 11\right)$, which may be caused by the unsteady natural of the force and moment in post-lock-in range.

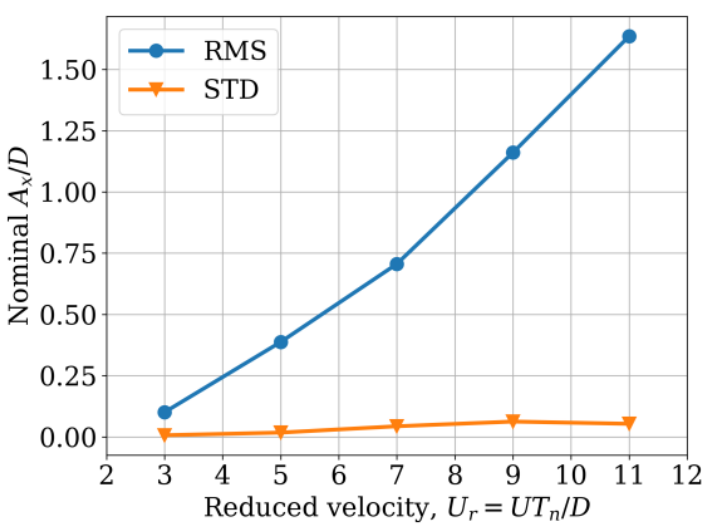

Figure 11: Nominal response of in-line motion

380 In similar, Figure 12 shows the nominal response of yaw motion. The nominal yaw motion is

381 monotonically increasing and reaches to about 2.55 degree at $U_{r}=11$. As mentioned previously, the 382 natural yaw period ( $\left.T_{n, \text { yaw }}=9.32 \mathrm{~s}\right)$ is much smaller than the natural transverse period $\left(T_{n}=15.45 \mathrm{~s}\right)$.

383 Obviously, yaw motion has a much higher natural frequency. Amongst the current cases, even the

384 highest reduced velocity does not reach the range which synchronization occurs between vortex 385 shedding and yaw motions. Redefining reduced velocity by yaw natural period $U_{r, \text { yaw }}=\frac{U T_{n, \text { yaw }}}{D}$, 
$U_{r}=11$ is corresponding to $U_{r, \text { yaw }}=6.6$, which is exactly the lock-in range in terms of yaw. We have no reason to doubt that as current velocity continually increasing, the nominal yaw response may still increase but eventually decrease when it comes to the post-lock-in range in terms of yaw.

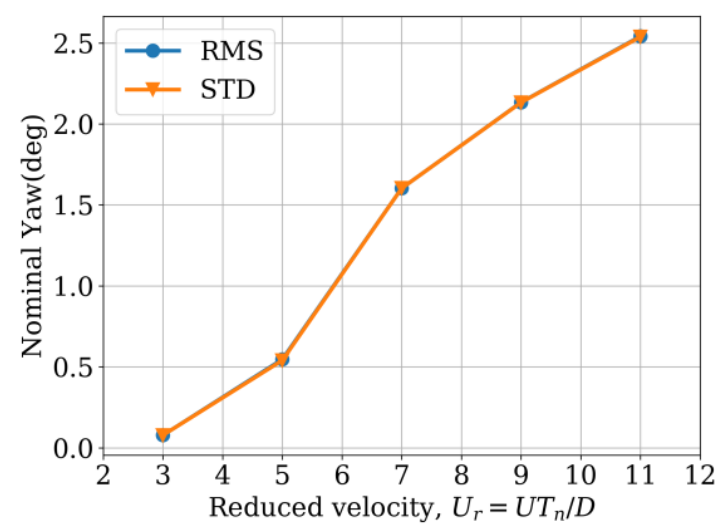

Figure 12: Nominal response of yaw motion

To better understanding the motion characteristics at different reduced velocities, further spectral analysis are performed for transverse, in-line and yaw motions. Figure 13 depicts the time history and FFT spectral analysis results for transverse motion at different reduced velocities. At low reduced velocity $\left(U_{r}=3\right)$, the small and irregular motion response with multiple frequencies suggest a motion state before lock-in. After entering lock-in range, the transverse motion is characterized by a dominant frequency which can be clearly seen in Figure 13(b)-(d). This confirms the strong modulated transverse motion in lock-in range. Unlike VIV, in which the shedding frequency is locked on one natural frequency in a wide range of reduced velocities (see for example [29]), the motion frequency of semi-submersible does not lock on one particular frequency. Instead, it increases as the increasing of reduced velocity. This may be attributed to the complex hull geometry (e.g., the multi-column structure and pontoon). 

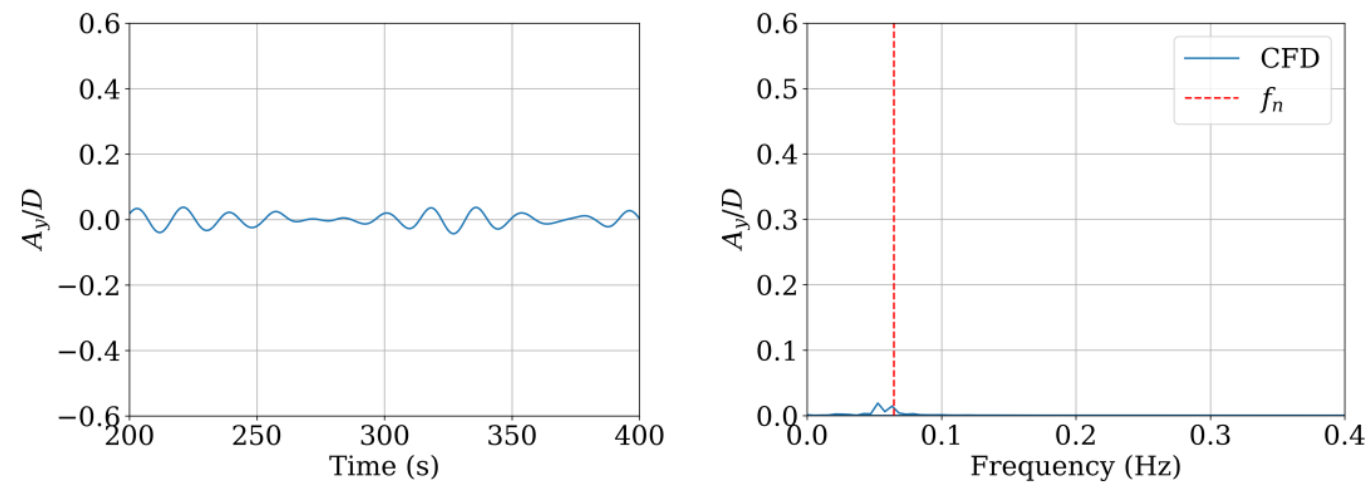

(a) $U_{r}=3$
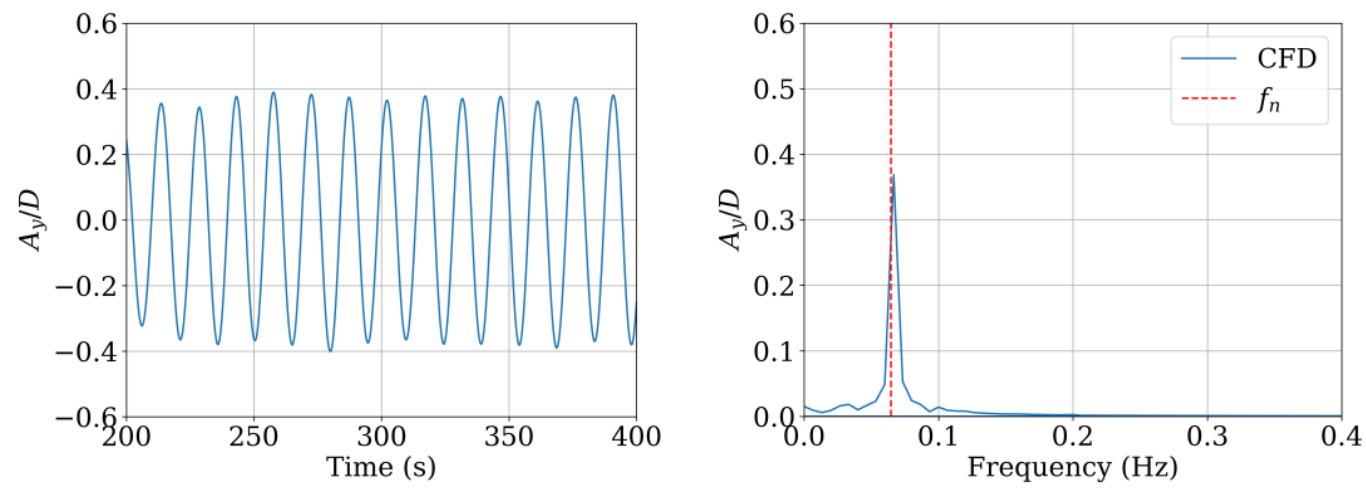

(b) $U_{r}=5$
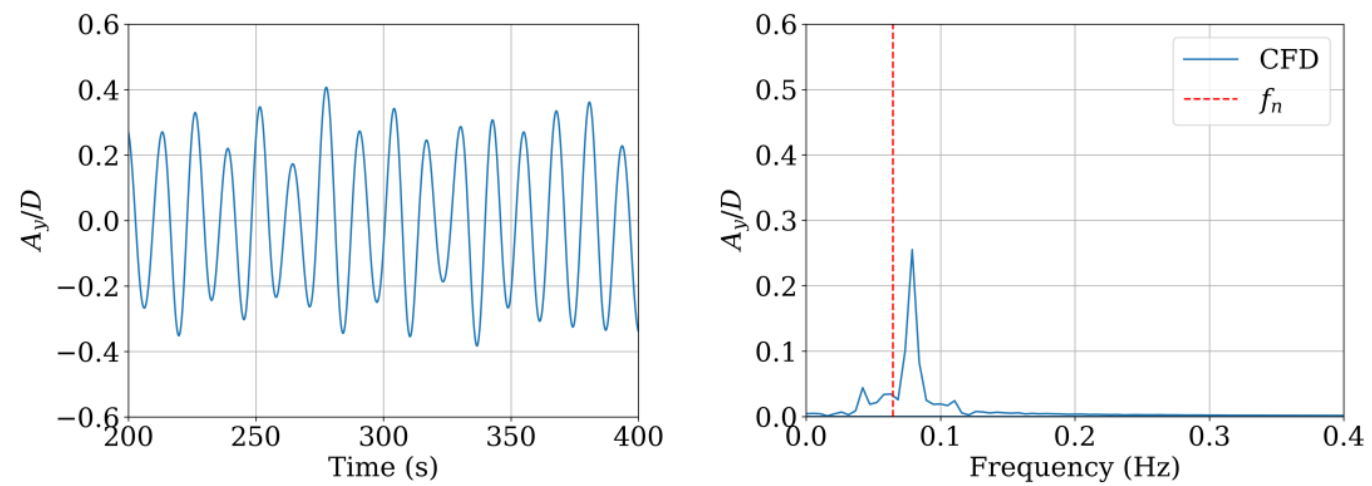

(c) $U_{r}=7$ 

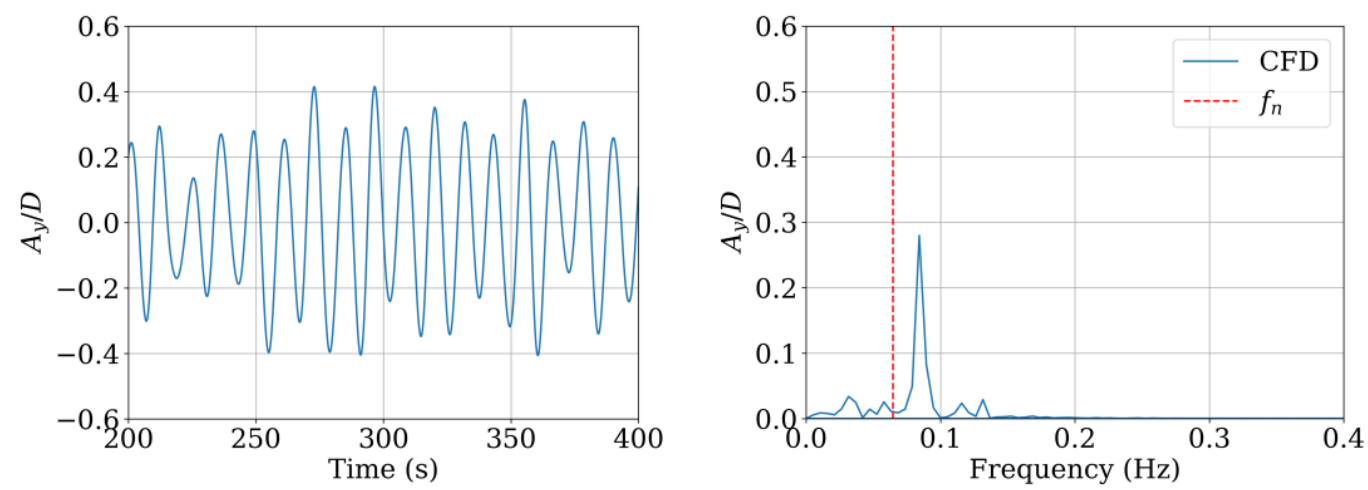

(d) $U_{r}=9$

Figure 13: Time history and spectral analysis of transverse motion

Similar to transverse motion, the time history and FFT spectral analysis for yaw motion are detailed in Figure 14. At pre-lock-in regime (e.g., $U_{r}=3$ ), yaw is fluctuating at small amplitude like transverse motion. When entering lock-in range $\left(U_{r}=5\right)$, dominant frequency occurs and characterizes the yaw motion. It should be emphasized that as the reduced velocity continually increases, a second dominant frequency appears near yaw natural frequency. The first peak frequency is undoubtedly the consequence of vortex shedding, as it is coincidence with the transverse motion frequency at corresponding velocity. This corroborates that the yaw motion of semi-submersible is induced by vortex shedding. The phenomena was termed vortex-induced yaw or VIY [30]. The occurrence of the second dominant frequency is induced by the interaction, particularly the synchronization between yaw motion and vortex shedding. This was previously reported by Gonçalves et al [30] reported in their model test of a large-volume semi-submersible platform. The existence of VIY shows the importance of yaw motions together with transverse motions in the VIM study of semi-submersibles. It also increases the difficulty and complexity to estimate the fatigue failure of risers and mooring system for semi-submersibles. 

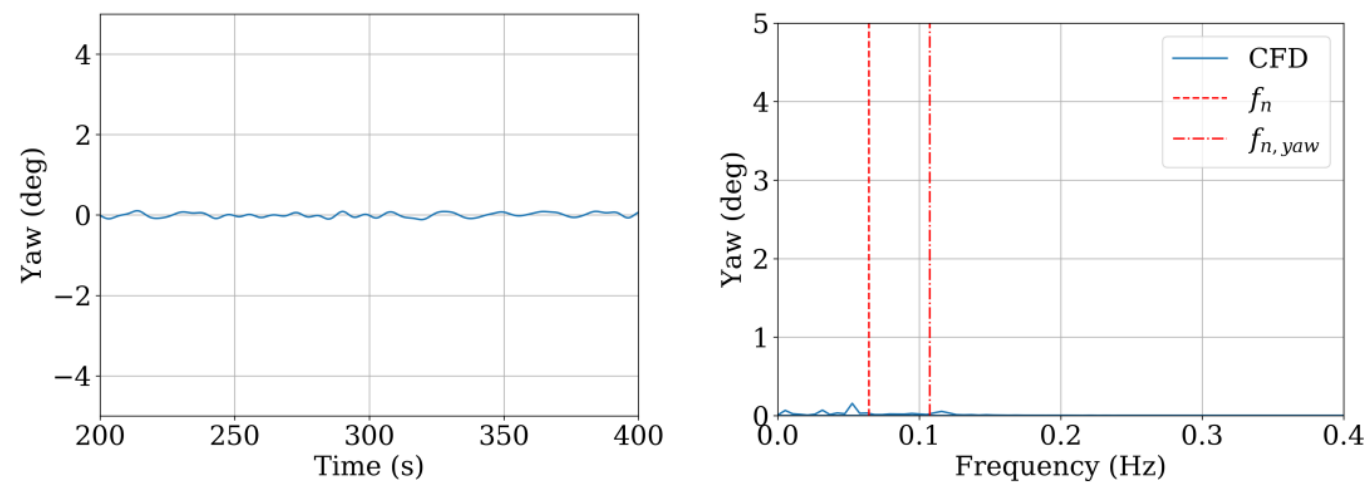

(a) $U_{r}=3$
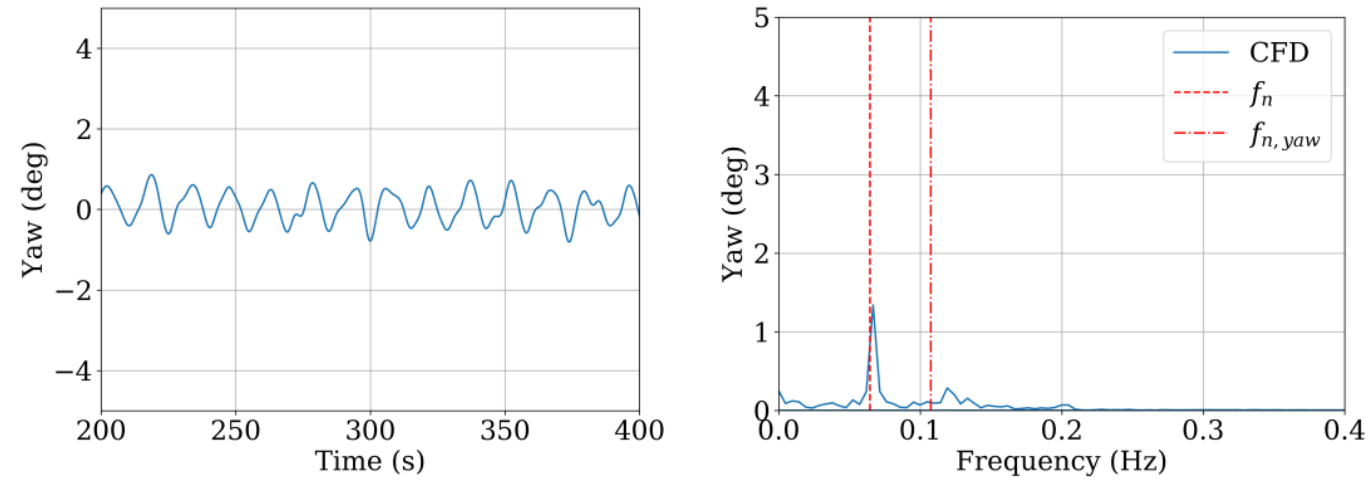

(b) $U_{r}=5$
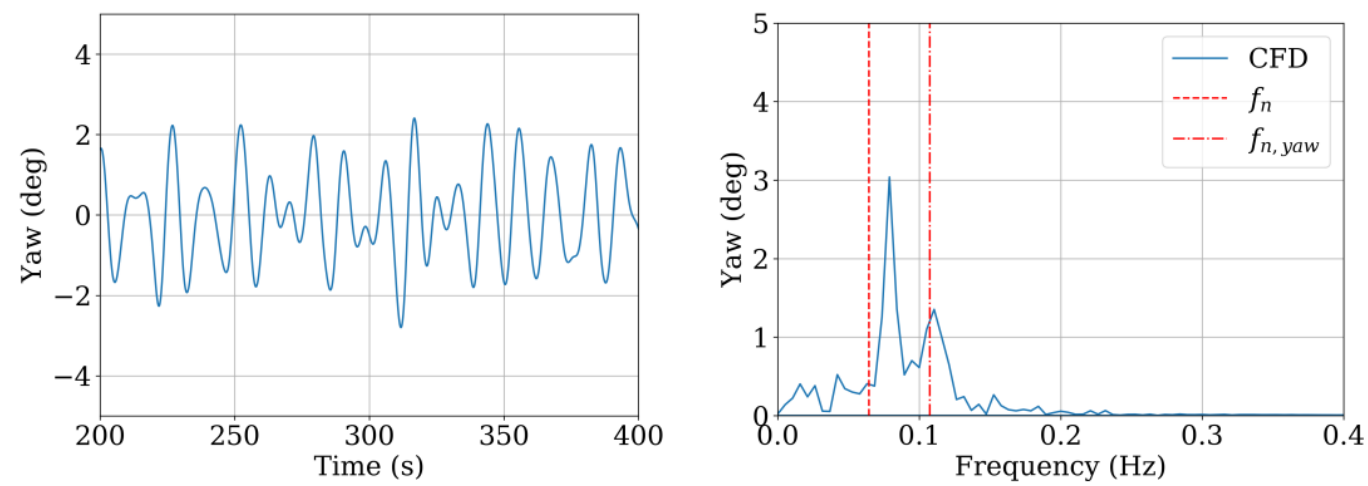

(c) $U_{r}=7$ 

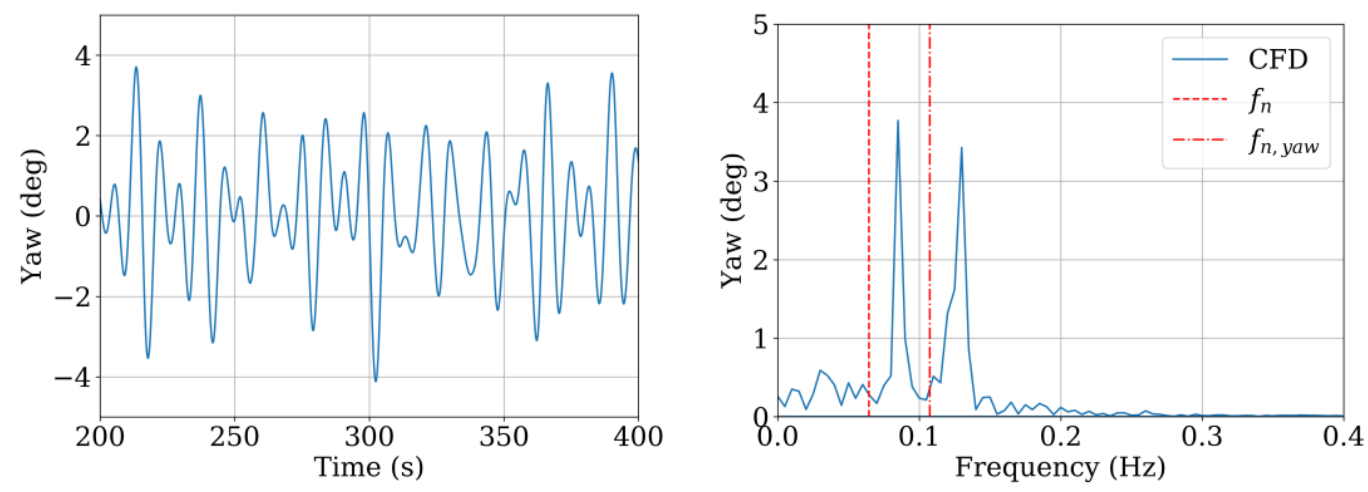

(d) $U_{r}=9$

Figure 14: Time history and spectral analysis of yaw motion

409

Figure 15 plots the motion trajectories of the hull centroid on horizontal plane. No typical eight (8)

shape trajectory is observed for all conditions. In lock-in range, the synchronized behavior results in pronounced transverse motion amplitude. The transverse motion response in post-lock-in range is the same magnitude to that in lock-in range. However, the in-line response fluctuates much stronger in pre-lock-in range. Trajectories at higher reduced velocities become more erratic.

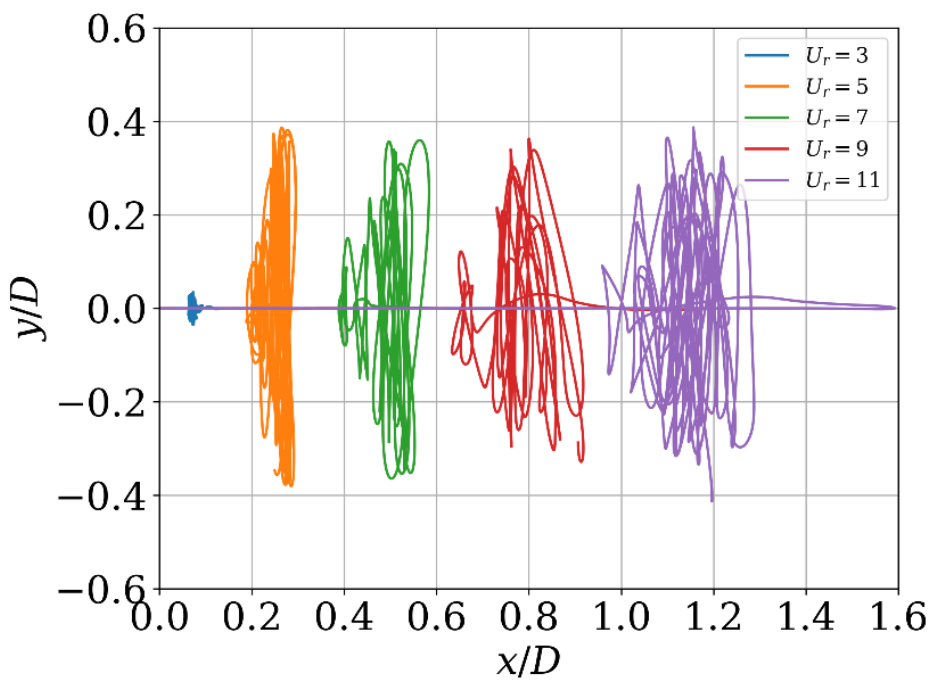

Figure 15: Motion trajectories of centroid at different reduced velocities

To analysis the effect of columns and pontoon on VIM characteristic, the work done by each 
412 component during stabilized VIM are calculated and presented in Figure 16. The work done is

413 calculated with the following formula [31]

$$
\begin{aligned}
& W_{x}=\int F_{x}(t) \cdot \dot{x}(t) d t \\
& W_{y}=\int F_{y}(t) \cdot \dot{y}(t) d t \\
& W=W_{x}+W_{y}
\end{aligned}
$$
wake interaction in this unique design of paired-column hull structure, as the work done transition

where $W_{x}$ and $W_{y}$ is work done in the in-line and transverse directions, respectively. $W$ is total work done. $F_{x}(t)$ and $F_{y}(t)$ are hydrodynamic force on each component in the in-line and For convenience, the columns are labeled by numbers. The definition can be found in Figure 7. Overall the work done by pontoon is negative for all reduced velocities. The magnitude of negative work done by pontoon are much larger than a single column, suggesting that pontoon could effectively mitigate VIM response. At low reduced velocity $\left(U_{r}=5\right)$, the excitations of VIM are mainly from upstream columns (OC \#1 and \#2, IC \#1 and \#2). The downstream OCs damp VIM a little and ICs have nearly no effects on VIM. However, this is not always the case. The work done by two upstream OCs turn from positive to negative at high reduced velocity $\left(U_{r}=9\right)$.

On the contrary, the work done by other columns except two upstream OCs are all positive. The reason for this change is unclear yet. A possible explanation may be attributed to the complex from positive to negative did not observed in previous study of conventional four-column semi-submersibles [23,31]. 


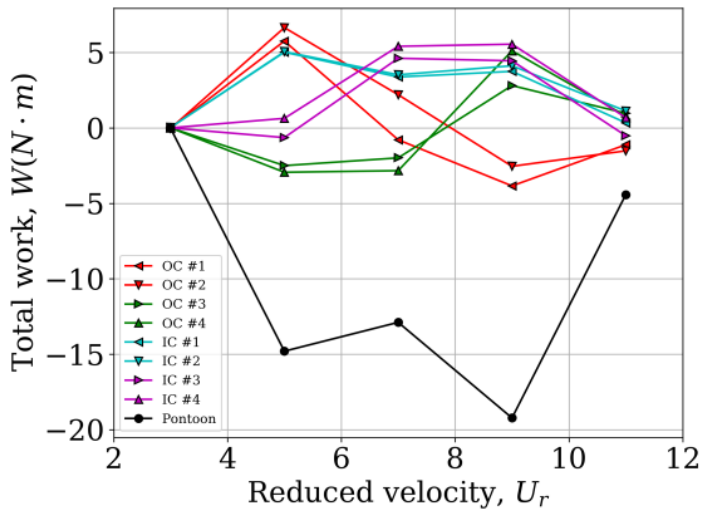

432 Figure 17 presents the instantaneous spanwise vorticity contour at different reduced velocities.

The vorticity is non-dimensionalized by the characteristic length and current velocity. The vortex

434 shedding mainly occurs at two lateral rounded corners for each upstream column. Vortices shed 435 from upstream column directly impinge on the downstream column, then collide and interact with

436 the vortices shed from downstream columns. These vortices quickly break into small eddies in the wake region of downstream columns. As $U_{r}$ increases to 5, VIM is entering lock-in scenario, the

438 vortex shedding patterns changes distinctively. Synchronized vortex shedding patterns are clearly

439 observed amongst the four upstream columns. Moreover, the vortices generated from two lateral

440 rounded corner are reattaching to the backface of each upstream column after flow separation.

441 This dramatically increases the hydrodynamic force and motion amplitude in transverse direction. 
442 Figure 17: Instantaneous non-dimensional spanwise vorticity contour at half draft $(z / H=-0.5)$ plane

\section{5. Conclusions}

444 Stationary drag and VIM simulations of a paired-column semi-submersible at model scale are

445 performed using an in-house CFD solver naoe-FOAM-SJTU. The turbulence flow is modeled

446 with SST-DES method and the motions are obtained by solving 6DoF equations. Dynamic overset grid is used to prevent the near wall mesh distortion during large yaw motions. Results from 
448 stationary drag simulations show that the current DES turbulence is applicable to accurately predict the drag of complex multi-column hull geometry. Several reduced velocities range from 3 to 11 are investigated for VIM. The transverse motion responses predict by the current numerical approach are in good agreement with CFD results by Antony et al. [24]. Spectral analysis using FFT for transverse and yaw motion time series are conducted. The transverse motion in lock-in scenario is governed by dominant frequency equal to vortex shedding frequency. Unlike VIV, the vortex shedding frequency in VIM of semi-submersible does not lock on one natural transverse frequency. It increases as current velocity increases. FFT results for yaw response show that yaw motion is induced by vortex shedding. Synchronized behavior for yaw motion occurs when shedding frequency is approaching yaw natural frequency. The work done by pontoon is always negative, suggesting the damping effect of pontoon on VIM response. The flow reattachment on the backface of upstream columns, together with the synchronized vortex shedding between multiple upstream columns, are accounting for the pronounced VIM motion in lock-in range.

\section{Acknowledgement}

This work is supported by the National Natural Science Foundation of China (51490675, 11432009, 51579145), Chang Jiang Scholars Program (T2014099), Shanghai Excellent Academic Leaders Program (17XD1402300), Program for Professor of Special Appointment (Eastern Scholar) at Shanghai Institutions of Higher Learning (2013022), Innovative Special Project of Numerical Tank of Ministry of Industry and Information Technology of China (2016-23/09) and Lloyd's Register Foundation for doctoral student, to which the authors are most grateful. 


\section{References}

471 [1] Dijk RRT van, Fourchy P, Voogt A, Mirza S. The effect of mooring system and sheared currents on vortex induced motions of truss Spars. Proc. 22nd Int. Conf. Offshore Mech. Arct. Eng., vol. 1, Cancun, Mexico: 2003, p. 285-92.

[2] Finnigan T, Roddier D. Spar VIM model tests at supercritical reynolds numbers. Proc. 26th Int. Conf. Offshore Mech. Arct. Eng., vol. 3, San Diego, California, USA: 2007, p.

[3] Roddier D, Finnigan T, Liapis S. Influence of the Reynolds number on spar Vortex 
Int. Ocean Polar Eng. Conf., Kona, Big Island, Hawaii, USA: 2015, p. 1048-55.

[8] Kim SJ, Spernjak D, Holmes S, Vinayan V, Antony A. Vortex-Induced Motion of Floating Structures: CFD Sensitivity Considerations of Turbulence Model and Mesh Refinement. Proc. ASME 2015 34th Int. Conf. Ocean Offshore Arct. Eng., vol. 2, St. John's, Newfoundland, Canada: 2015, p. V002T08A057.

[9] Chen C-R, Chen H-C. Simulation of vortex-induced motions of a deep draft semi-submersible in current. Ocean Eng 2016;118:107-16.

[10] Kara M, Kaufmann J, Gordon R, Sharma P, Lu J. Application of CFD for Computing VIM of Floating Structures. Offshore Technol. Conf., Houston, Texas, USA: 2016.

[11] Vinayan V, Antony A, Halkyard J, Kim S-J, Holmes S, Spernjak D. Vortex-induced motion of deep-draft semisubmersibles: A CFD-based parametric study. Proc. ASME 2015 34th Int. Conf. Ocean Offshore Arct. Eng., vol. 2, St. John's, Newfoundland, Canada: 2015, p. V002T08A003.

[12] Rosetti GF, Gonçalves R, Fujarra ALC, Koop A. CFD calculations of the vortex-induced motions of a circular-column semi-submersible. Proc. ASME 2016 35th Int. Conf. Ocean Offshore Arct. Eng., vol. 2, Busan, Korea: 2016.

[13] Zhao W, Shen Z, Wan D. Numerical Investigation of the Vortex Induced Motion of SPAR in Uniform Current. Proc. 24th Int. Ocean Polar Eng. Conf., vol. 3, Busan, Korea: ISOPE; 2014, pp. 362-7.

[14] Gordon R, Mostofi R. ULTRA-DEEPWATER DRY TREE SYSTEM FOR DRILLING AND PRODUCTION IN THE GULF OF MEXICO. Final Technical Report, 
10121-4405-02, RPSEA-Research Partnership to Secure Energy for America, Report; 2014.

513

Shen Z, Wan D. RANS computations of added resistance and motions of a ship in head waves. Int J Offshore Polar Eng 2013; 23: 263-71.

[16] Shen Z, Wan D, Carrica PM. Dynamic overset grids in OpenFOAM with application to KCS self-propulsion and maneuvering. Ocean Eng., 2015; 108: 287-306.

[17] Noack RW. SUGGAR: A general capability for moving body overset grid assembly. 17th AIAA Comput. Fluid Dyn. Conf., Toronto, Ontario, Canada: 2005.

[18] Noack RW, Boger DA, Kunz RF, Carrica PM. Suggar++: An improved general overset grid assembly capability. 19th AIAA Comput. Fluid Dyn. Conf., San Antonio, Texas, USA: 2009.

[19] Cao H, Wan D. Application of OpenFOAM to simulate three-dimensional flows past a single and two tandem circular cylinders. Proc. Int. Offshore Polar Eng. Conf., vol. 3, 2010, pp. 702-9.

[20] Zhou H, Cao H, Wan D. Numerical Predictions of Wave Impacts on the Supporting Structures of Shanghai Donghai-Bridge Offshore Wind Turbines. Twenty-Third Int. Offshore Polar Eng. Conf., Anchorage, Alaska, USA: International Society of Offshore and Polar Engineers; 2013.

[21] Wang J, Zou L, Wan D. CFD simulations of free running ship under course keeping control. Ocean Eng 2017;141:450-64.

[22] Liu M, Xiao L, Yang J, Tian X. Parametric study on the vortex-induced motions of 
semi-submersibles: Effect of rounded ratios of the column and pontoon. Phys Fluids 2017;29:055101.

[23] Liang Y, Tao L. Interaction of vortex shedding processes on flow over a deep-draft

[24] Antony A, Vinayan V, Holmes S, Spernjak D, Kim SJ, Halkyard J. VIM Study for Deep Draft Column Stabilized Floaters. Offshore Technol. Conf., Houston, Texas, USA: 2015.

[25] Waals OJ, Phadke AC, Bultema S. Flow induced motions of multi column floaters. Proc. 26th Int. Conf. Offshore Mech. Arct. Eng., vol. 1, San Diego, California, USA: 2007, p. $669-78$.

26] Rijken O, Leverette S. Experimental Study Into Vortex Induced Motion Response of Semi Submersibles With Square Columns. Proc. ASME 27th Int. Conf. Offshore Mech. Arct. Eng., vol. 1, Estoril, Portugal: 2008, p. 263-76.

[27] Gonçalves RT, Nishimoto K, Rosetti GF, Fujarra ALC, Oliveira AC. Experimental study on vortex-induced motions (VIM) of a large-volume semi-submersible platform. Proc. ASME 2011 30th Int. Conf. Ocean Offshore Arct. Eng., vol. 7, Rotterdam, The Netherlands: 2011, pp. 1-9.

[28] Zou J, Poll P, Roddier D, Tom N, Peiffer A. VIM testing of a paired column semi submersible. Proc. ASME 2013 32nd Int. Conf. Ocean Offshore Arct. Eng., vol. 7, Nantes, France: 2013, p. V007T08A001.

[29] KHALAK A, WILLIAMSON CHK. MOTIONS, FORCES AND MODE TRANSITIONS IN VORTEX-INDUCED VIBRATIONS AT LOW MASS-DAMPING. J Fluids Struct 
553

554

555

556

557

558

559

560

1999;13:813-51.

[30] Gonçalves RT, Rosetti GF, Fujarra C, Luís A, Nishimoto K, Oliveira C. Vortex-Induced Yaw Motion (VIY) of a Large-Volume Semi-Submersible Platform, Rhodes, Greece: International Society of Offshore and Polar Engineers; 2012.

[31] Liu M, Xiao L, Liang Y, Tao L. Experimental and numerical studies of the pontoon effect on vortex-induced motions of deep-draft semi-submersibles. J Fluids Struct 2017; 72: 59-79. 